\title{
Applications of a model for silicon resonant sensors
}

\author{
Albert Prak and Jan H J. Fluttman \\ MESA Research Instutute, Universuty of Twente, PO Bax 217, 7500 AE Enschede (Netherlands)
}

\begin{abstract}
A recently developed model for excitation and detection mechanisms of silicon resonant sensors is experimentally verified by applying it to practical devices Various system aspects of resonators with piezoelectric and electrostatic excitation/detection come into focus The experimental results agree reasonably well with simulation results obtained by the model
\end{abstract}

\section{Introduction}

Silicon resonant sensors $[1,2]$ are suitable for measurement of mechanical quantities (force, pressure, acceleration, etc) with high precision The operating principle of silicon resonant sensors is that the resonance frequency of a micromachined slicon structurc (for example, a prismatic beam) shifts as the measurand changes The resonance frequency is the sensor output signal Recently, we developed a systematic unifying model to describe the excitation and detection mechanisms of this sensor family [3] The model is based on a system approach the resonator, including the transducers for excitation/detection is regarded as a full electromechanical system In ref 3 , the model is derived, and a comparative review of excitation and detection mechanisms for micromechanical resonators is given In this paper, we demonstrate the practical applicability of the model

\section{The sensor model}

In order to activate and sense the vibration, one or more electromechanical transducers for excitation and detection have to be incorporated in the sensor system For this purpose, various mechanisms are suitable Here, we restrict ourselves to mechanisms of the reversible type Energy can be exchanged with the system through the transducers The energy flow through a single transducer is charactenzed by an untensive variable (e g, a voltage $u$ ) and a corresponding extensive vanable (e g, a charge $q$ ) The power flow equals the product of the intensive variable and the time derivative of the extensive variable, which is called extensity flow variable, or just flow Every transducer serves as a power port of the system The number of electrical ports of the system equals the number of electromechanical transducers

The system has also mechanical ports Sunce the mass, stiffness and damping of the resonator are distributed, an infinite number of mechanical ports is required An clegant discretization is obtaincd when the mechanics of the resonator is described in terms of its eigenstates, also called vibration modes In that case, the modal displacements $y_{t}$ are the extensive variables, and the modal forces $\boldsymbol{P}_{\boldsymbol{i}}$ are the intensive variables The modal approach is similar to a Fourier series the shape of the vibrating resonator is expanded in the mode shapes The number of mechanical ports of the system equals the number of modes taken into account Since resonant sensors are operated near resonance, good approxlmations can be obtained with only a few mechanical ports

Thus, the total system has $n+m$ energy ports, where $m$ stands for the number of electromechanical transducers and $n$ for the number of modes taken into account $A$ bond-graph representation of the sensor system is shown in Fig 1 Small harmonic variations around an equilibrium state can be described by the matrix equation

$$
\left|\begin{array}{l}
\bar{u} \\
\bar{P}
\end{array}\right|=\left|\begin{array}{ll}
\mathrm{E} & \mathrm{C} \\
\mathrm{C}^{\mathrm{T}} & \mathrm{M}
\end{array}\right|\left|\begin{array}{l}
\bar{q} \\
\bar{y}
\end{array}\right|
$$

where $\mathbf{E}, \mathbf{M}$ and $\mathbf{C}$ are an electrical, a mechanical and an electromechanical coupling matrix, respectively, $\bar{u}$ and $\dot{q}$ are vectors of length $m$, and $\dot{P}$ and $\bar{y}$ are vectors of length $n$ The dot indicates differentiation with respect to time and the overbar indicates amplitude Equation (1) is called the constituent equation of the system 


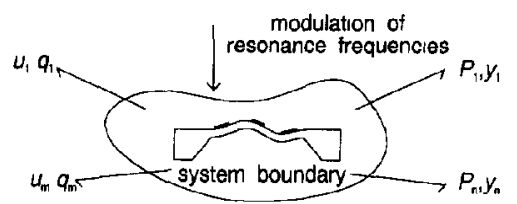

electrical ports

mechanıcal ports

Fig 1 Bond-graph representation of a resonant sensor with $m$ electromechanical transducers for excitation/detection purposes and $n$ vibration modes taken into account

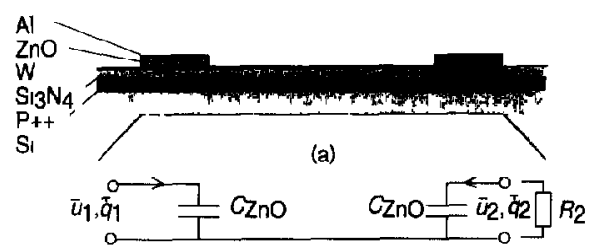

(b)

Fig 2 Cross section (a) and electrical scheme (b) of the piezoelectrically operated resonant force sensor

\section{Electrical transfer function of a piezoelectric resonator}

At the MESA Institute, research is performed on a piezoelectrically operated resonant force sensor [4] The sensor has two electromechanical transducers for excitation and detection The transducers consist of a thin piezoelectric $\mathrm{ZnO}$ film sandwiched between a tungsten and an aluminum electrode This three-layer structure is placed on top of the resonator (see Fig 2) Only the first two modes of vibration are taken into account Hence, the system model has two electrical and two mechanical ports

The mechanical submatrix $M$ of the constituent eqn (1) merely consists of the mechanical spring effect of the modes Off-diagonal elements of this matrix, as well as the electrical submatrix $\mathbf{E}$, are zero Diagonal elements of $E$ represent the impedance of the capacities of the $\mathrm{ZnO}$ layers Finally, the coupling matrix $\mathrm{C}$ reflects the electromechanical transducing capabilities of the excitation/detection elements Important parameters in the matrix elements of $\mathrm{C}$ are the piezoelectric coefficient, $d_{31}$ of the $\mathrm{ZnO}$, the intrinsic coupling factor of the $\mathrm{ZnO}$, the thickness of the prezoelectric film and a geometrical factor which is a measure of the efficiency of a transducer to couple energy to or from a specific mode For the exact detalls concerning the matrux elements we refer to refs 3 and 5

The system is described by eight variables four intensive and four flow variables To calculate the electrical transfer function, $1 \mathrm{e}$, the ratio $\bar{u}_{2} / \bar{u}_{1}$, seven equations are required Four equations are obtained from the constituent equation The other three are phenomenological equations They describe how the power ports are loaded or controlled by the outside world. The two mechanical ports are loaded by viscous friction forces (which can be obtained from modal quality factors) and inertia (the modal masses) The electrical port used for the detection is loaded by a resistance $R_{2}$ The excitation port is controlled by a voltage source

Simulation results of the transfer function $\bar{u}_{2} / \bar{u}_{1}$ are given in Fig 3 Simulations are performed for three values of $R_{2}$, namely, $R_{2}=01 R_{\text {char }}, R_{2}=R_{\text {char }}$ and $R_{2}=10 R_{\text {char }} R_{\text {char }}$ is the modulus of the impedance of the detection transducer at the first resonance frequency $R_{\text {char }}=1 / \omega_{1} C_{\mathrm{ZnO}}$, where $\omega_{1}$ is the angular resonance frequency of the first mode, and $C_{\mathrm{ZnO}}$ is the capacity of the $\mathrm{ZnO}$ transducer

The load impedance $R_{2}$ forms a high pass filter in combination with the capacity of the transducer $C_{\mathrm{Zno}}$ The turnover frequency of this inherent filter is given by $f_{\text {to }}=1 /\left(2 \pi C_{Z_{\text {no }}} R_{2}\right)$ The filter characteristic is clearly visible in Fig 3 If $R_{2} \ll R_{\text {char }}$ the resonance peaks are on the $20 \mathrm{~dB} /$ decade flank of the filter and the loop of the second mode is larger than that of the first mode For $R_{2} \gg R_{\text {char }}$, both resonance frequencies are higher than the turnover frequency and the loop of the first mode is the larger one Another observation is that the phase at resonance shifts from $0^{\circ}$ to $-90^{\circ}$ as the load impedance varies from $R_{2} \ll R_{\text {char }}$ to $R_{2} \gg R_{\text {char }}$ Considerations like this are of the utmost importance when designing feedback electronics for the oscillator

The above charactenstics have been experimentally verified Figure 4 shows the measured polar plots for frequencies around the first resonance frequency The rotational orientation and the sizes of the resonance loop are determined by position of the turnover frequency of the filter with respect to the first resonance frequency The aforementioned high-pass frequency characteristic manifests itself by a hemicycle in the first quadrant of the polar plot This is shown in Fig 5

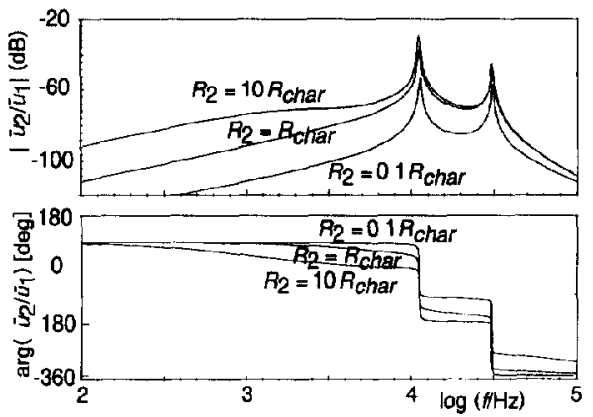

Fig 3 Bode diagram of the piezoelectric force sensor for three values of $\boldsymbol{R}_{2}$ 


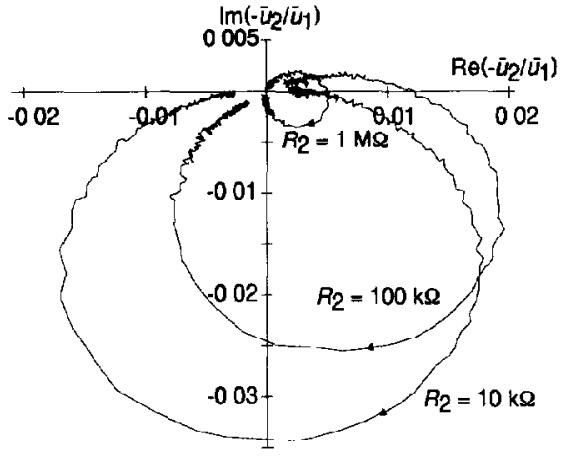

Fig 4 Measured polar plots of the transfer function of the piezoelectric force sensor for three values of $R_{2}$ The plots are for trequencies near $\omega_{1}$

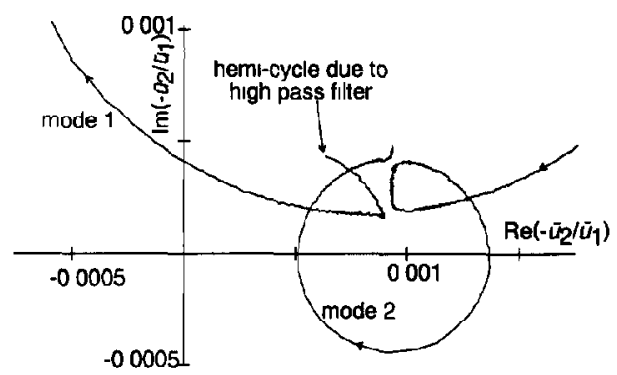

Fig 5 Measured polar plot of the first two resonances of the piezoelectric force sensor The Figure also shows the last part of a hemicycle, which is a consequence of the inherent high pass filter

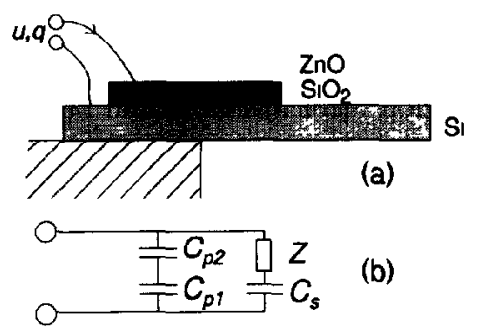

Fig 6 Cross section (a) and electrical scheme (b) of the test structure for determination of piezoelectric properties

\section{Characternzation of piezoelectric materials}

The sensor model can be exploited quantitatively to detcrmine piczoelectnc propertics of $\mathrm{ZnO}$ films using the resonance method For this purpose, a cantilever resonator with one $\mathrm{ZnO}$ transducer (see Fig 6) was used The device can be modelled as a system with one electrical and one mechanical port (one mode is taken into account)
The impedance of this device, measured at the electrical port, can be written as

$Z(\omega)=\frac{\bar{u}}{\bar{q}}=\frac{1}{j \omega C_{1}}+\frac{1}{j \omega C_{2}} \frac{1}{1+\mathrm{J} \frac{\omega}{\omega_{1}} \frac{1}{Q_{1}}-\frac{\omega^{2}}{\omega_{1}^{2}}}$

where the constants $C_{1}$ and $C_{2}$ are composed of piezoelectric, electric and mechanical properties of $\mathrm{ZnO}$ and the dimensions/shape of the transducer and the resonator $C_{1}$ and $C_{2}$ can be obtained from the matrix elements of the constituent equation For the exact expressions of $C_{1}$ and $C_{2}$ we refer to refs 3 and 5

The first term of eqn (2) results in a straight line extending along the negative imaginary axis of the polar impedance plot (see Fig 7) The second term only contributes near resonance and results in a loop Properties of the $\mathrm{ZnO}$ can be obtaned from the diameter of this loop, provided that the dimensions of the structure and its $Q$ factor are known $\emptyset_{\text {loop }} \alpha\left(d_{31} / \epsilon_{33} s_{11}\right)^{2}$

The relative permittivity $\epsilon_{33}$ can be determined by a capacitance measurement The compliance $s_{11}$ and $d_{31}$ cannot be separated without performing a pure mechanical measurement The ratio $d_{31} / \epsilon_{33} s_{11}$ can be interpreted as a figure of ment for piezoelectrically operated resonators

Two samples were measured A typical impedance measurement is shown in Fig 7 It is noteworthy that the measured drameter of the loop was corrected for the series and parallel capacitances, which are the result of an $\mathrm{SiO}_{2}$ layer and the extension of the film on the substrate, respectively (see Fig 6) Table 1 summarizes the results Literature values of bulk $\mathrm{ZnO}$ are also

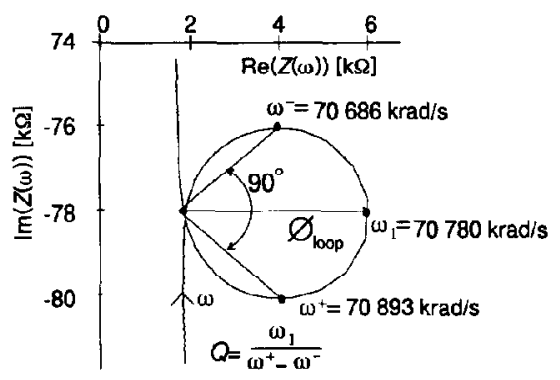

Fig 7 Polar impedance plot of the test structure

TABLE 1 Material properties of sputtered $\mathrm{ZnO}$ film

\begin{tabular}{lcll}
\hline Sample & $\epsilon_{33} / \epsilon_{0}$ & $\begin{array}{l}d_{31} / s_{11} \\
\left(\mathrm{C} / \mathrm{m}^{2}\right)\end{array}$ & $\begin{array}{l}d_{31} \\
(\mathrm{PC} / \mathrm{N})\end{array}$ \\
\hline I & 9 & 019 & $-15^{\mathrm{a}}$ \\
II & 9 & 025 & $-20^{5}$ \\
bulk & 113 & 064 & -51 \\
\hline
\end{tabular}

'Value determined using the $s_{11}$ value of bulk $\mathrm{ZnO}$ 
given The $\mathrm{ZnO}$ was sputtered with a substrate temperature of $425^{\circ} \mathrm{C}$, an $\mathrm{RF}$ power of $1800 \mathrm{~W}$ for $1 \mathrm{~min}$, and $1000 \mathrm{~W}$ for the remainder of the time $(2 \mathrm{~h})$ The oxygen pressure was $05 \mathrm{~Pa}$ The thickness of the film was $368 \mu \mathrm{m}$ It was assumed that the whole film shows a homogeneous piezoelectric activity

\section{Loaded Qs}

Since high $Q$ resonant sensors generally have a high resolution, a high $Q$ is usually pursued The $Q$ of the resonance, being defined as the ratio of the stored vibration energy and the periodically dissipated energy, is often determined by mechanical considerations However, energy can be dissipated not only at mechanical ports, but also at electrical ports by resistively loading the transducers In this manner, it is possible that a resonator exhibits a $Q$ which is considerably lower than the $Q$ based on mechanical considerations

The $Q$, based on dissipation in the electrical domain, of a resonator system with one vibration mode and one transducer is given by [3]

$Q_{\mathrm{el}}=\frac{1}{k^{2}} \frac{\left|1+\mathbf{E} / Z_{\text {load }}\right|}{|\mathbf{E}| \operatorname{Re}\left(1 / Z_{\text {load }}\right)}$

where the electromechanical coupling factor, defined as $k^{2}=\mathrm{C}^{2} / \mathrm{ME}$, was supposed small The minimum $Q_{\mathrm{el}}$ is obtained for $Z_{\text {load }}=|\mathbf{E}|$ and equals $Q_{\mathrm{cl}, \min }=2 / k^{2}$ At this load impedance, maximum signal-to-noise ratio is obtained In most cases, the coupling factor is rather small and $Q_{\mathrm{c} 1}$ is overruled by the $Q$ based on mechanical considerations For example, the piezoelectric force sensor (Section 3) has a coupling factor $k=001$, which makes $Q_{\mathrm{el} \mathrm{min}}=2 \times 10^{4}$ However, it is well known that the coupling factor of a resonator driven by an electrostatic air-gap mechanism [3] can be vaned from 0 to 1 by increasing the bias voltage from 0 to the pullin voltage This is shown in Fig 8

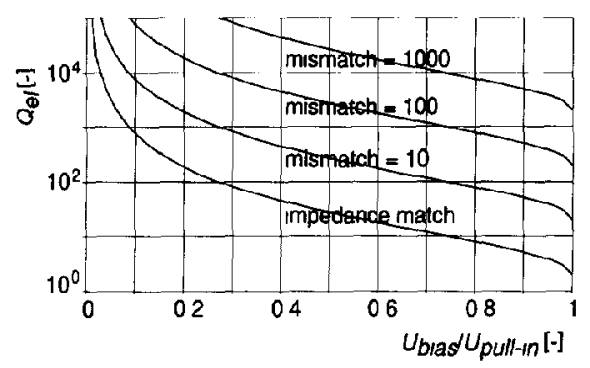

Fig $8 Q_{\mathrm{el}}$ as a function of the ratio $u_{\text {bas }} / u_{\text {pul an }}$ for various values of the impedance mismatch $|E| / Z_{\text {bad }}$ The mechanical $Q$ was assumed infinite and $Z_{\text {lond }}$ was assumed real (system loaded resistively)
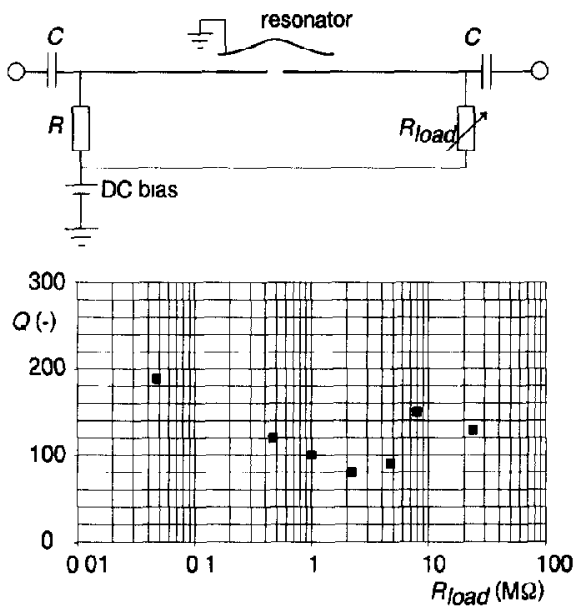

Fig 9 Experimental set-up (top) and measurement results (bottom) showing the effect of an electric load on the system's quality factor

Figure 9 shows experimental results of a electrostatically operated resonator, the load impedance of which was vaned Clearly, the measured $Q$ is limited by dissipation in the electric domain (the mechanically based $Q$ was approximately 500 ) The bias voltage was set at $90 \%$ of the pull-in voltage, resulting in a coupling factor $k=07$ This would imply a minimum $Q_{\mathrm{el}}=$ $2 / k^{2}=5$, however, due to parasitic capactances the load is not purely resistive A complex load results in a higher $Q_{\text {el min }}[5]$

\section{Conclusions}

Excitation and detection mechanisms should be regarded as integral parts of silicon resonant sensor systems A recently developed model has been verified by various experiments, emphasizing system aspects The model appears to be a powerful tool to describe the functioning of silicon resonant sensors and their interaction with the environment

\section{Acknowledgements}

The authors are grateful to Cees van Mullem and Gert-Jan Burger for making avallable their measurement results

\section{References}

1 G Stemme, Resonant sulicon sensors, J Mucromech Microeng, $I$ (1991) 113-125 
2 H A C Tulmans, M Elwenspoek and J H J Fluitman, Micro resonant force gauges, Sensors and Actuators $A, 30$ (1992) 35-53

3 A Prak, T S J Lammerınk and J H J Fluitman, Revew of excitation and detection mechanisms for micromechanical resonators, Sensors Mater, accepted for publication
4 C J van Mullem, FR Blom, J H J Flustman and $M$ Elwenspoek, Piezoelectrically driven silicon beam force sensor, Sensors and Actuators A, 25-27 (1991) 379. 383

5 A Prak, Silicon resonant sensors operation and response, Ph $D$ Thesis, Unversity of Twente, 1993 